\title{
Learned-Norm Pooling for Deep Feedforward and Recurrent Neural Networks
}

\author{
Caglar Gulcehre, Kyunghyun Cho, Razvan Pascanu, and Yoshua Bengio* \\ Département d'Informatique et de Recherche Opérationelle \\ Université de Montréal
}

$(\star)$ CIFAR Fellow

\begin{abstract}
In this paper we propose and investigate a novel nonlinear unit, called $L_{p}$ unit, for deep neural networks. The proposed $L_{p}$ unit receives signals from several projections of a subset of units in the layer below and computes a normalized $L_{p}$ norm. We notice two interesting interpretations of the $L_{p}$ unit. First, the proposed unit can be understood as a generalization of a number of conventional pooling operators such as average, root-mean-square and max pooling widely used in, for instance, convolutional neural networks (CNN), HMAX models and neocognitrons. Furthermore, the $L_{p}$ unit is, to a certain degree, similar to the recently proposed maxout unit [13] which achieved the state-of-the-art object recognition results on a number of benchmark datasets. Secondly, we provide a geometrical interpretation of the activation function based on which we argue that the $L_{p}$ unit is more efficient at representing complex, nonlinear separating boundaries. Each $L_{p}$ unit defines a superelliptic boundary, with its exact shape defined by the order $p$. We claim that this makes it possible to model arbitrarily shaped, curved boundaries more efficiently by combining a few $L_{p}$ units of different orders. This insight justifies the need for learning different orders for each unit in the model. We empirically evaluate the proposed $L_{p}$ units on a number of datasets and show that multilayer perceptrons (MLP) consisting of the $L_{p}$ units achieve the state-of-the-art results on a number of benchmark datasets. Furthermore, we evaluate the proposed $L_{p}$ unit on the recently proposed deep recurrent neural networks (RNN).
\end{abstract}

Keywords: deep learning, $L_{p}$ unit, multilayer perceptron

\section{Introduction}

The importance of well-designed nonlinear activation functions when building a deep neural network has become more apparent recently. Novel nonlinear activation functions that are unbounded and often piecewise linear but not continuous such as rectified linear units (ReLU) 22[11], or rectifier, and maxout units [13] have been found to be particularly well suited for deep neural networks on many object recognition tasks.

A pooling operator, an idea which dates back to the work in [17, has been adopted in many object recognizers. Convolutional neural networks which often 
employ max pooling have achieved state-of-the-art recognition performances on various benchmark datasets [209]. Also, biologically inspired models such as HMAX have employed max pooling [27. A pooling operator, in this context, is understood as a way to summarize a high-dimensional collection of neural responses and produce features that are invariant to some variations in the input (across the filter outputs that are being pooled).

Recently, the authors of [13] proposed to understand a pooling operator itself as a nonlinear activation function. The proposed maxout unit pools a group of linear responses, or outputs, of neurons, which overall acts as a piecewise linear activation function. This approach has achieved many state-of-the-art results on various benchmark datasets.

In this paper, we attempt to generalize this approach by noticing that most pooling operators including max pooling as well as maxout units can be understood as special cases of computing a normalized $L_{p}$ norm over the outputs of a set of filter outputs. Unlike those conventional pooling operators, however, we claim here that it is beneficial to estimate the order $p$ of the $L_{p}$ norm instead of fixing it to a certain predefined value such as $\infty$, as in max pooling.

The benefit of learning the order $p$, and thereby a neural network with $L_{p}$ units of different orders, can be understood from geometrical perspective. As each $L_{p}$ unit defines a spherical shape in a non-Euclidean space whose metric is defined by the $L_{p}$ norm, the combination of multiple such units leads to a non-trivial separating boundary in the input space. In particular, an MLP may learn a highly curved boundary efficiently by taking advantage of different values of $p$. In contrast, using a more conventional nonlinear activation function, such as the rectifier, results in boundaries that are piece-wise linear. Approximating a curved separation of classes would be more expensive in this case, in terms of the number of hidden units or piece-wise linear segments.

In Sec. 2 a basic description of a multi-layer perceptron (MLP) is given followed by an explanation of how a pooling operator may be considered a nonlinear activation function in an MLP. We propose a novel $L_{p}$ unit for an MLP by generalizing pooling operators as $L_{p}$ norms in Sec. 3. In Sec. 4 the proposed $L_{p}$ unit is further analyzed from the geometrical perspective. We describe how the proposed $L_{p}$ unit may be used by recurrent neural networks in Sec. 5. Sec. 6 provides empirical evaluation of the $L_{p}$ unit on a number of object recognition tasks.

\section{Background}

\subsection{Multi-layer Perceptron}

A multi-layer perceptron (MLP) is a feedforward neural network consisting of multiple layers of nonlinear neurons 29. Each neuron $u_{j}$ of an MLP typically receives a weighted sum of the incoming signals $\left\{a_{1}, \ldots, a_{N}\right\}$ and applies a non- 
linear activation function $\phi$ to generate a scalar output such that

$$
u_{j}\left(\left\{a_{1}, \ldots, a_{N}\right\}\right)=\phi\left(\sum_{i=1}^{N} w_{i j} a_{i}\right) .
$$

With this definition of each neuron ${ }^{1}$, we define the output of an MLP having $L$ hidden layers and $q$ output neurons given an input $\mathbf{x}$ by

$$
\mathbf{u}(\mathbf{x} \mid \boldsymbol{\theta})=\phi\left(\mathbf{U}^{\top} \phi_{[L]}\left(\mathbf{W}_{[L]}^{\top} \cdots \phi_{[1]}\left(\mathbf{W}_{[1]}^{\top} \mathbf{x}\right) \cdots\right)\right),
$$

where $\mathbf{W}_{[l]}$ and $\phi_{[l]}$ are the weights and the nonlinear activation function of the $l$-th hidden layer, and $\mathbf{W}_{[1]}$ and $\mathbf{U}$ are the weights associated with the input and output, respectively.

\subsection{Pooling as a Nonlinear Unit in MLP}

Pooling operators have been widely used in convolutional neural networks (CNN) 211027. to reduce the dimensionality of a high-dimensional output of a convolutional layer. When used to group spatially neighboring neurons, this operator which summarizes a group of neurons in a lower layer is able to achieve the property of (local) translation invariance. Various types of pooling operator have been proposed and used successfully, such as average pooling, root-of-mean-squared (RMS) pooling and max pooling 19133.

A pooling operator may be viewed instead as a nonlinear activation function. It receives input signal from the layer below, and it returns a scalar value. The output is the result of applying some nonlinear function such as max (max pooling). The difference from traditional nonlinearities is that the pooling operator is not applied element-wise on the lower layer, but rather on groups of hidden units. A maxout nonlinear activation function proposed recently in [13] is a representative example of $\max$ pooling in this respect.

\section{$3 \quad L_{p}$ Unit}

The recent success of maxout has motivated us to consider a more general nonlinear activation function that is rooted in a pooling operator. In this section, we propose and discuss a new nonlinear activation function called an $L_{p}$ unit which replaces the max operator in a maxout unit by an $L_{p}$ norm.

\subsection{Normalized $L_{p}$-norm}

Given a finite vector/set of input signals $\left[a_{1}, \ldots, a_{N}\right]$ a normalized $L_{p}$ norm is defined as

$$
u_{j}\left(\left[a_{1}, \ldots, a_{N}\right]\right)=\left(\frac{1}{N} \sum_{i=1}^{N}\left|a_{i}-c_{i}\right|^{p_{j}}\right)^{\frac{1}{p_{j}}},
$$

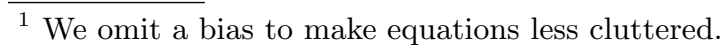




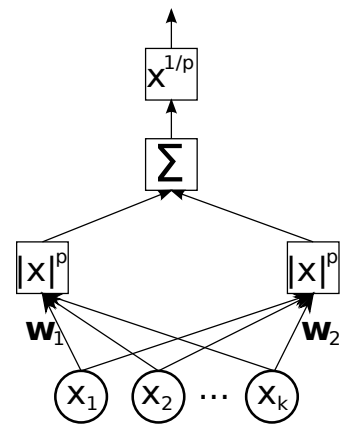

(a)

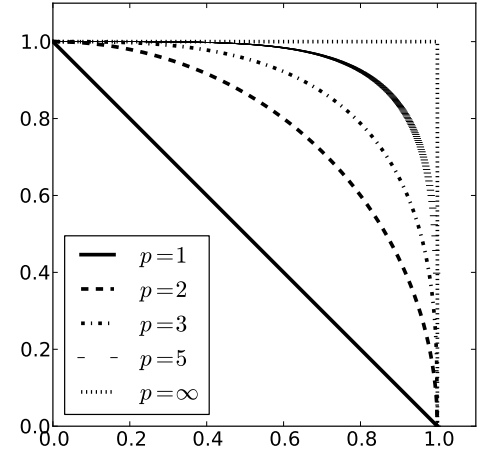

(b)

Fig. 1. (a) An illustration of a single $L_{p}$ unit with two sets of incoming signals. For clarity, biases and the division by the number of filters are omitted. The symbol $x$ in each block (square) represents an input signal to that specific block (square) only. (b) An illustration of the effect of $p$ on the shape of an ellipsoid. Only the first quadrant is shown.

where $p_{j}$ indicates that the order of the norm may differ for each neuron. It should be noticed that when $0<p_{j}<1$ this definition is not a norm anymore due to the violation of triangle inequality. In practice, we re-parameterize $p_{j}$ by $1+\log \left(1+e^{\rho_{j}}\right)$ to satisfy this constraint.

The input signals (also called filter outputs) $a_{i}$ are defined by

$$
a_{i}=\mathbf{w}_{i}^{\top} \mathbf{x}
$$

where $\mathbf{x}$ is a vector of activations from the lower layer. $c_{i}$ is a center, or bias, of the $i$-th input signal $a_{i}$. Both $p_{j}$ and $c_{i}$ are model parameters that are learned.

We call a neuron with this nonlinear activation function an $L_{p}$ unit. An illustration of a single $L_{p}$ unit is presented in Fig. 1 (a).

Each $L_{p}$ unit in a single layer receives input signal from a subset of linear projections of the activations of the layer immediately below. In other words, we project the activations of the layer immediately below linearly to $A=\left\{a_{1}, \ldots, a_{N}\right\}$. We then divide $A$ into equal-sized, non-overlapping groups of which each is fed into a single $L_{p}$ unit. Equivalently, each $L_{p}$ unit has its private set of filters.

The parameters of an MLP having one or more layers of $L_{p}$ units can be estimated by using backpropagation [30, and in particular we adapt the order $p$ of the norm ${ }^{2}$ In our experiments, we use Theano [5] to compute these partial derivatives and update the orders $p_{j}$ (through the parametrization of $p_{j}$ in terms of $\rho_{j}$ ), as usual with any other parameters.

2 The activation function is continuous everywhere except a finite set of points, namely when $a_{i}-c_{i}$ is 0 and the absolute value function becomes discontinuous. We ignore these discontinuities, as it is done, for instance, in maxouts and rectifiers. 


\subsection{Related Approaches}

Thanks to the definition of the proposed $L_{p}$ unit based on the $L_{p}$ norm, it is straightforward to see that many previously described nonlinear activation functions or pooling operators are closely related to or special cases of the $L_{p}$ unit. Here we discuss some of them.

When $p_{j}=1$, Eq. (3) becomes

$$
u_{j}\left(\left[a_{1}, \ldots, a_{N}\right]\right)=\frac{1}{N} \sum_{i=1}^{N}\left|a_{i}\right| .
$$

If we further assume $a_{i} \geq 0$, for instance, by using a logistic sigmoid activation function on the projection of the lower layer, the activation is reduced to computing the average of these projections. This is a form of average pooling, where the non-linear projections represent the pooled layer. With a single filter, this is equivalent to the absolute value rectification proposed in [19]. If $p_{j}$ is 2 instead of 1 , the root-of-mean-squared pooling from 33 is recovered.

As $p_{j}$ grows and ultimately approaches $\infty$, the $L_{p}$ norm becomes

$$
\lim _{p_{j} \rightarrow \infty} u_{j}\left(\left[a_{1}, \ldots, a_{N}\right]\right)=\max \left\{\left|a_{1}\right|, \ldots,\left|a_{N}\right|\right\} .
$$

When $N=2$, this is a generalization of a rectified linear unit (ReLU) as well as the absolute value unit [19. If each $a_{i}$ is constrained to be non-negative, this corresponds exactly to the maxout unit.

In short, the proposed $L_{p}$ unit interpolates among different pooling operators by the choice of its order $p_{j}$. This was noticed earlier in [8] as well as [33]. However, both of them stopped at analyzing the $L_{p}$ norm as a pooling operator with a fixed order and comparing those conventional pooling operators against each other. The authors of [4] investigated a similar nonlinear activation function that was inspired by the cells in the primary visual cortex. In [18], the possibility of learning $p$ has been investigated in a probabilistic setting in computer vision.

On the other hand, in this paper, we claim that the order $p_{j}$ needs to, and can be learned, just like all other parameters of a deep neural network. Furthermore, we conjecture that (1) an optimal distribution of the orders of $L_{p}$ units differs from one dataset to another, and (2) each $L_{p}$ unit in a MLP requires a different order from the other $L_{p}$. These properties also distinguish the proposed $L_{p}$ unit from the conventional radial-basis function network (see, e.g., [15])

\section{Geometrical Interpretation}

We analyze the proposed $L_{p}$ unit from a geometrical perspective in order to motivate our conjecture regarding the order of the $L_{p}$ units. Let the value of an $L_{p}$ unit $u$ be given by:

$$
u(\mathbf{x})=\left(\frac{1}{N} \sum_{i=1}^{N}\left|\mathbf{w}_{i}^{\top} \mathbf{x}-c_{i}\right|^{p}\right)^{\frac{1}{p}},
$$




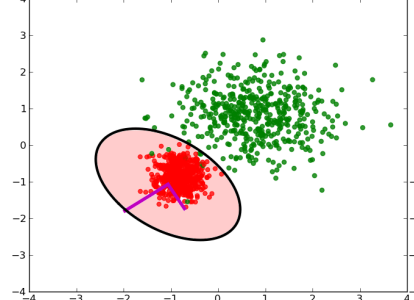

(a) $L_{p}$ with $p=2$

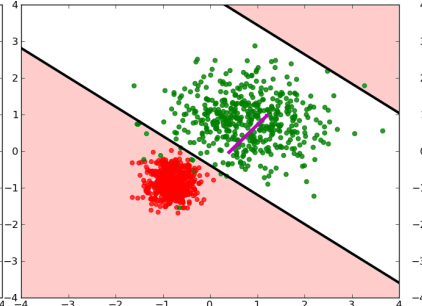

(b) $L_{p}$ with $p=\infty$

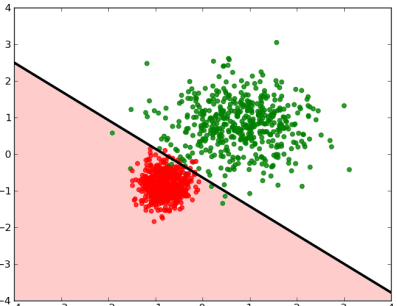

(c) Rectifier

Fig. 2. Visualization of separating curves obtained using different activation functions. The underlying data distribution is a mixture of two Gaussian distributions. The red and green dots are the samples from the two classes, respectively, and the black curves are separating curves found by the MLPs. The purple lines indicate the axes of the subspace learned by each $L_{p}$ unit. Best viewed in color.

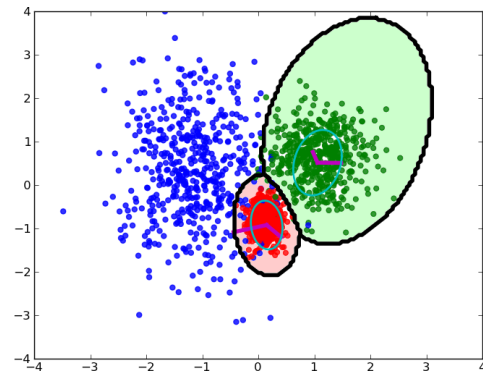

(a) $p_{1}=p_{2}=2$

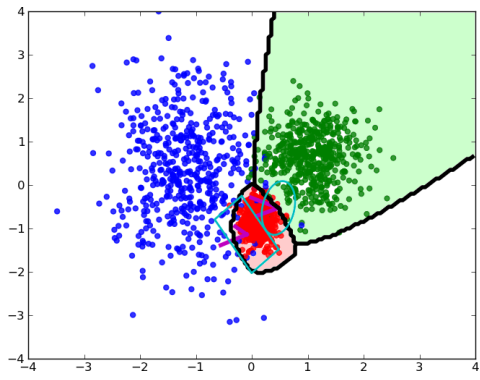

(b) $p_{1}=2$ and $p_{2}=\infty$

Fig. 3. Visualization of separating curves obtained using different orders of $L_{p}$ units. The underlying data distribution is a mixture of three Gaussian distributions. The blue curves show the shape of the superellipse learned by each $L_{p}$ unit. The red, green and blue dots are the samples. Otherwise, the same color convention as in Fig. 2 has been used.

where $\mathbf{w}_{i}$ represents the $i$-th column of the matrix $\mathbf{W}$. The equation above effectively says that the $L_{p}$ unit computes the $p$-th norm of the projection of the input $\mathbf{x}$ on the subspace spanned by $N$ vectors $\left\{\mathbf{w}_{1}, \ldots, \mathbf{w}_{N}\right\}$. Let us further assume that $\mathbf{x} \in \mathbb{R}^{d}$ is a vector in an Euclidean space.

The space onto which $\mathbf{x}$ is projected may be spanned by linearly dependent vectors $\mathbf{w}_{i}$ 's. Due to the possible lack of the linear independence among these vectors, they span a subspace $\mathcal{S}$ of dimensionality $k \leq N$. The subspace $\mathcal{S}$ has its origin at $\mathbf{c}=\left[c_{1}, \ldots, c_{N}\right]$.

We impose a non-Euclidean geometry on this subspace by defining a norm in the space to be $L_{p}$ with $p$ potentially not 2, as in Eq. (4). The geometrical object to which a particular value of the $L_{p}$ unit corresponds forms a superellipse when projected back into the original input space. ${ }^{3}$ The superellipse is centered at the

3 Since $k \leq N$, the superellipse may be degenerate in the sense that in some of the $N-k$ axes the width may become infinitely large. However, as this does not invalidate 
inverse projection of $\mathbf{c}$ in the Euclidean input space. Its shape varies according to the order $p$ of the $L_{p}$ unit and due to the potentially linearly-dependent bases. As long as $p \geq 1$ the shape remains convex. Fig. 1 (b) draws some of the superellipses one can get with different orders of $p$, as a function of $a_{1}$ (with a single filter).

In this way each $L_{p}$ unit partitions the input space into two regions - inside and outside the superellipse. Each $L_{p}$ unit uses a curved boundary of learned curvature to divide the space. This is in contrast to, for instance, a maxout unit which uses piecewise linear hyperplanes and might require more linear pieces to approximate the same curved segment.

\subsection{Qualitative Analysis in Low Dimension}

When the dimensionality of the input space is 2 and each $L_{p}$ receives 2 input signals, we can visualize the partitions of the input space obtained using $L_{p}$ units as well as conventional nonlinear activation functions. Here, we examine some artificially generated cases in a 2-D space.

Two Classes, Single $\boldsymbol{L}_{\boldsymbol{p}}$ Unit Fig. 2 shows a case of having two classes (• and $\bullet$ ) of which each corresponds to a Gaussian distribution. We trained MLPs having a single hidden neuron. When the MLPs had an $L_{p}$ unit, we fixed $p$ to either 2 or $\infty$. We can see in Fig. 2 (a) that the MLP with the $L_{2}$ unit divides the input space into two regions - inside and outside a rotated superellipse ${ }^{4}$ The superellipse correctly identified one of the classes (red).

In the case of $p=\infty$, what we see is a degenerate rectangle which is an extreme form of a superellipse. The superellipse again spotted one of the classes and appropriately draws a separating curve between the two classes.

In the case of rectifier units it could find a correct separating curve, but it is clear that a single rectifier unit can only partition the input space linearly unlike $L_{p}$ units. A combination of several rectifier units can result in a nonlinear boundary, specifically a piecewise-linear one, though our claim is that you need more such rectifier units to get an arbitrarily shaped curve whose curvature changes in a highly nonlinear way.

Three Classes, Two $\boldsymbol{L}_{p}$ Units Similarly to the previous experiment, we trained two MLPs having two $L_{p}$ units on data generated from a mixture of three Gaussian distribution. Again, each mixture component corresponds to each class.

For one MLP we fixed the orders of the two $L_{p}$ units to 2. In this case, see Fig. 3 (a), the separating curves are constructed by combining two translated

our further argument, we continue to refer this kind of (degenerate) superellipse simply by an superellipse.

4 Even though we use $p=2$, which means an Euclidean space, we get a superellipse instead of a circle because of the linearly-dependent bases $\left\{\mathbf{w}_{1}, \ldots, \mathbf{w}_{N}\right\}$. 
superellipses represented by the $L_{p}$ units. These units were able to locate the two classes, which is sufficient for classifying the three classes $(\bullet, \bullet$ and $\bullet)$.

The other MLP had two $L_{p}$ units with $p$ fixed to 2 and $\infty$, respectively. The $L_{2}$ unit defines, as usual, a superellipse, while the $L_{\infty}$ unit defines a rectangle. The separating curves are constructed as a combination of the translated superellipse and rectangle and may have more non-trivial curvature as in Fig. 3 (b).

Furthermore, it is clear from the two plots in Fig. 3 that the curvature of the separating curves may change over the input space. It will be easier to model this non-stationary curvature using multiple $L_{p}$ units with different $p$ 's.

Decision Boundary with Non-Stationary Curvature: Representational Efficiency In order to test the potential efficiency of the proposed $L_{p}$ unit from its ability to learn the order $p$, we have designed a binary classification task that has a decision boundary with a non-stationary curvature. We use 5000 data points of which a subset is shown in Fig. 4 (a), where two classes are marked with blue dots $(\bullet)$ and red crosses $(+)$, respectively.

On this dataset, we have trained MLPs with either $L_{p}$ units, $L_{2}$ units $\left(L_{p}\right.$ units with fixed $p=2$ ), maxout units, rectifiers or logistic sigmoid units. We varied the number of parameters, which correspond to the number of units in the case of rectifiers and logistic sigmoid units and to the number of inputs signals to the hidden layer in the case of $L_{p}$ units, $L_{2}$ units and maxout units, from 2 to 16 . For each setting, we trained ten randomly initialized MLPs. In order to reduce effects due to optimization difficulties, we used in all cases natural conjugate gradient 23 .

From Fig. 4 (c), it is clear that the MLPs with $L_{p}$ units outperform all others in terms of representing this specific curve. They were able to achieve the zero training error with only three units (i.e., 6 filters) on all ten random runs and achieved the lowest average training error even with less units. Importantly, the comparison to the performance of the MLPs with $L_{2}$ units shows that it is beneficial to learn the orders $p$ of $L_{p}$ units. For example, with only two $L_{2}$ units none of the ten random runs succeed while at least one succeeds with two $L_{p}$ units. All the other MLPs, especially ones with rectifiers and maxout units which can only model the decision boundary with piecewise linear functions, were not able to achieve the similar efficiency of the MLPs with $L_{p}$ units (see Fig 4 (b)).

Fig. 4 (a) also shows the decision boundary found by the MLP with two $L_{p}$ units after training. As can be observed from the shapes of the $L_{p}$ units (purple and cyan dashed curves), each $L_{p}$ unit learned an appropriate order $p$ that enables them to model the non-stationary decision boundary. Fig. 4 (b) shows the boundary obtained by a rectifier model with four units. We can see that it has to use linear segments to compose the boundary, resulting in not perfectly solving the task. The rectifier model represented here has 64 mistakes, versus 0 obtained by the $L_{p}$ model. 


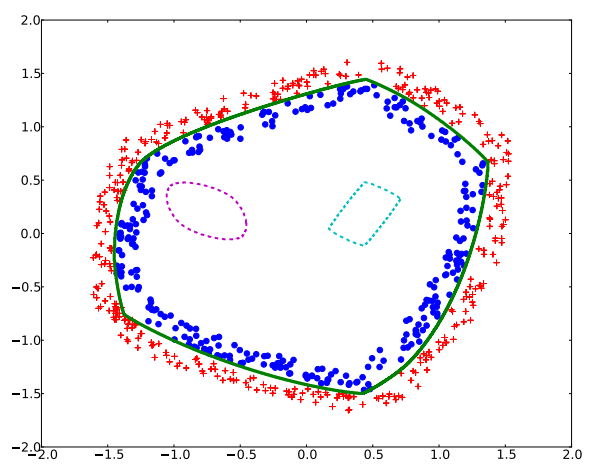

(a)

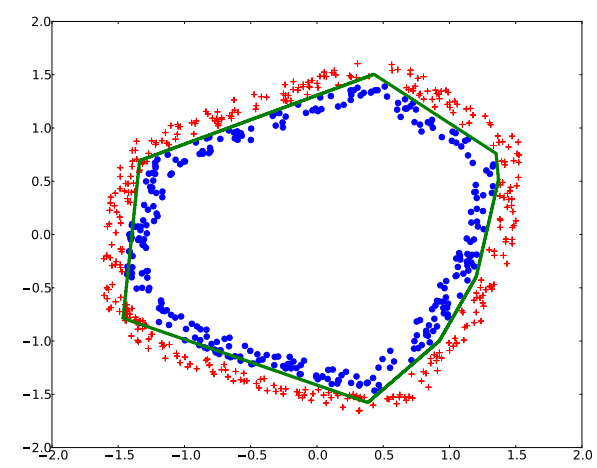

(b)

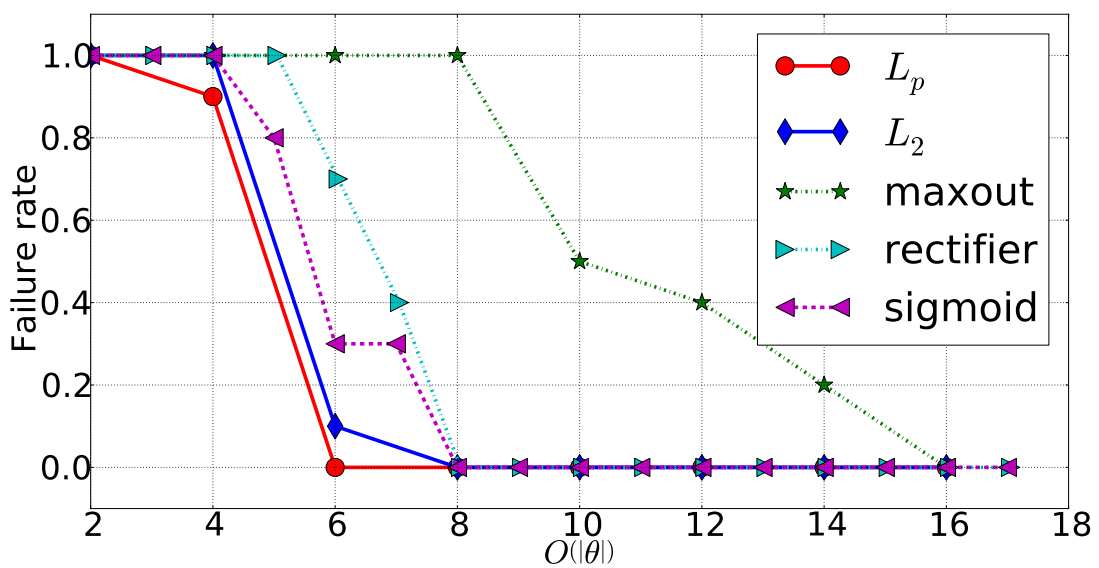

(c)

Fig. 4. (a) Visualization of data (two classes, + and $\bullet$ ), a decision boundary learned by an MLP with two $L_{p}$ units (green curve) and the shapes corresponding to the orders $p$ 's learned by the $L_{p}$ units (purple and cyan dashed curves). (b) The same visualization done using four rectifiers. (c) The failure rates computed with MLPs using different numbers of different nonlinear activation functions $\left(L_{p}\right.$ : red solid curve with red $\bullet$, $L_{2}$ : blue solid curve with blue $\downarrow$, maxout: green dashed curve with green $\star$, rectifier: cyan dash-dot curve with cyan $>$ and sigmoid: purple dashed curve with purple 4). The curves show the proportion of the failed attempts over ten random trials (y-axis) against either the number of units for sigmoid and rectifier model or the total number of linear projection going into the maxout units or $L_{p}$ units (x-axis). 


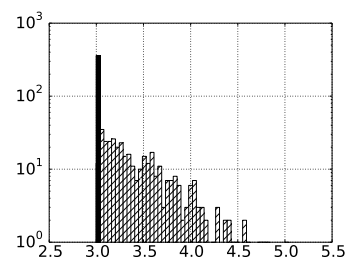

(a) MNIST

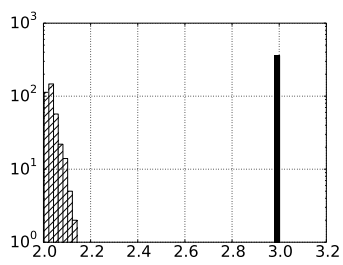

(b) TFD

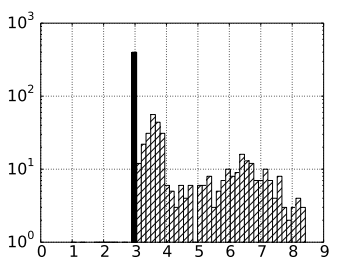

(c) Pentomino

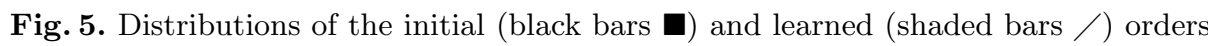
on MNIST, TFD and Pentomino. $x$-axis and $y$-axis show the order and the number of $L_{p}$ units with the corresponding order. Note the difference in the scales of the x-axes and that the y-axes are in logarithmic scale.

Although this is a low-dimensional, artificially generated example, it demonstrates that the proposed $L_{p}$ units are efficient at representing decision boundaries which have non-stationary curvatures.

\section{Application to Recurrent Neural Networks}

A conventional recurrent neural network (RNN) mostly uses saturating nonlinear activation functions such as tanh to compute the hidden state at each time step. This prevents the possible explosion of the activations of hidden states over time and in general results in more stable learning dynamics. However, at the same time, this does not allow us to build an RNN with recently proposed non-saturating activation functions such as rectifiers and maxout as well as the proposed $L_{p}$ units.

The authors of [24] recently proposed three ways to extend the conventional, shallow RNN into a deep RNN. Among those three proposals, we notice that it is possible to use non-saturating activations functions for a deep RNN with deep transition without causing the instability of the model, because a saturating non-linearity (tanh) is applied in sandwich between the $L_{p}$ MLP associated with each step.

The deep transition RNN (DT-RNN) has one or more intermediate layers between a pair of consecutive hidden states. The transition from a hidden state $\mathbf{h}_{t-1}$ at time $t-1$ to the next hidden state $\mathbf{h}_{t}$ is

$$
\mathbf{h}_{t}=g\left(\mathbf{W}^{\top} f\left(\mathbf{U}^{\top} \mathbf{h}_{t-1}+\mathbf{V}^{\top} \mathbf{x}_{t}\right)\right),
$$

not showing biases, as previously.

When a usual saturating nonlinear activation function is used for $g$, the activations of the hidden state $\mathbf{h}_{t}$ are bounded. This allows us to use any, potentially non-saturating nonlinear function for $f$. We can simply use a layer of the proposed $L_{p}$ unit in the place of $f$.

As argued in 24], if the procedure of constructing a new summary which corresponds to the new hidden state $\mathbf{h}_{t}$ from the combination of the current 
input $\mathbf{x}_{t}$ and the previous summary $\mathbf{h}_{t-1}$ is highly nonlinear, any benefit of the proposed $L_{p}$ unit over the existing, conventional activation functions in feedforward neural networks should naturally translate to these deep RNNs as well. We show this effect empirically later by training a deep output, deep transition RNN (DOT-RNN) with the proposed $L_{p}$ units.

\section{$6 \quad$ Experiments}

In this section, we provide empirical evidences showing the advantages of utilizing the $L_{p}$ units. In order to clearly distinguish the effect of employing $L_{p}$ units from introducing data-specific model architectures, all the experiments in this section are performed by neural networks having densely connected hidden layers.

\subsection{Claims to Verify}

Let us first list our claims about the proposed $L_{p}$ units that need to be verified through a set of experiments. We expect the following from adopting $L_{p}$ units in an MLP:

1. The optimal orders of $L_{p}$ units vary across datasets

2. An optimal distribution of the orders of $L_{p}$ units is not close to a (shifted) Dirac delta distribution

The first claim states that there is no universally optimal order $p_{j}$. We train MLPs on a number of benchmark datasets to see the resulting distribution of $p_{j}$ 's. If the distributions had been similar between tasks, claim 1 would be rejected.

This naturally connects to the second claim. As the orders are estimated via learning, it is unlikely that the orders of all $L_{p}$ units will convergence to a single value such as $\infty$ (maxout or max pooling), 1 (average pooling) or 2 (RMS pooling). We expect that the response of each $L_{p}$ unit will specialize by using a distinct order. The inspection of the trained MLPs to confirm the first claim will validate this claim as well.

On top of these claims, we expect that an MLP having $L_{p}$ units, when the parameters including the orders of the $L_{p}$ units are well estimated, will achieve highly competitive classification performance. In addition to classification tasks using feedforward neural networks, we anticipate that a recurrent neural network benefits from having $L_{p}$ units in the intermediate layer between the consecutive hidden states, as well.

\subsection{Datasets}


For feedforward neural networks or MLPs, we have used four datasets; MNIST [21, Pentomino 14, the Toronto Face Database (TFD) 31 and Forest Covertyp 5 (data split DS2-581) 32. MNIST, TFD and Forest Covertype are three representative benchmark datasets, and Pentomino is a relatively recently proposed dataset that is known to induce a difficult optimization challenge for a deep neural network. We have used three music datasets from [7] for evaluating the effect of $L_{p}$ units on deep recurrent neural networks.

\subsection{Distributions of the Orders of $L_{p}$ Units}

To understand how the estimated orders $p$ of the proposed $L_{p}$ unit are distributed we trained MLPs with a single $L_{p}$ layer on MNIST, TFD and Pentomino. We measured validation error to search for good hyperparameters, including the number of $L_{p}$ units and number of filters (input signals) per $L_{p}$ unit. However, for Pentomino, we simply fixed the size of the $L_{p}$ layer to 400, and each $L_{p}$ unit received signals from six hidden units below.

In Table 1, the averages and standard deviations of the estimated orders of the $L_{p}$ units in the single-layer MLPs are listed for MNIST, TFD and Pentomino. It is clear that the distribution of the orders depend heavily on the dataset, which confirms our first claim described earlier. From Fig. 5 we can clearly see that even in a single model the estimated orders vary quite a lot, which confirms our second claim. Interestingly, in the case of Pentomino, the distribution of the orders consists of two distinct modes.

The plots in Fig. 5 clearly show that the orders of the $L_{p}$ units change significantly from their initial values over training. Although we initialized the orders of the $L_{p}$ units around 3 for all the datasets, the resulting distributions of the orders are significantly different among those three datasets. This further confirms both of our claims. As a simple empirical confirmation we tried the same experiment with the fixed $p=2$ on TFD and achieved a worse test error of 0.21 .

\subsection{Generalization Performance}

The ultimate goal of any novel nonlinear activation function for an MLP is to achieve better generalization performance. We conjectured that by learning the orders of $L_{p}$ units an MLP with $L_{p}$ layers will achieve highly competitive classification performance.

For MNIST we trained an MLP having two $L_{p}$ layers followed by a softmax output layer. We used a recently introduced regularization technique called

\footnotetext{
${ }^{5}$ We use the first 16 principal components only.
} 


\begin{tabular}{r|cccc}
\hline Data & MNIST & TFD & \multicolumn{2}{c}{ Pentomino Forest Covertype } \\
\hline \hline$L_{p}$ & $0.97 \%$ & $20.75 \%$ & $31.85 \%$ & $2.83 \%$ \\
\hline Previous & $0.94 \%^{1}$ & $21.29 \%^{2}$ & $44.6 \%^{3}$ & $2.78 \%^{4}$ \\
\hline
\end{tabular}

Table 2. The generalization errors on three datasets obtained by MLPs using the proposed $L_{p}$ units. The previous state-of-the-art results obtained by others are also presented for comparison.

dropout [16. With this MLP we were able to achieve $99.03 \%$ accuracy on the test set, which is comparable to the state-of-the-art accuracy of $99.06 \%$ obtained by the MLP with maxout units [13].

On TFD we used the same MLP from the previous experiment to evaluate generalization performance. We achieved a recognition rate of $79.25 \%$. Although we use neither pretraining nor unlabeled samples, our result is close to the current state-of-the-art rate of $82.4 \%$ on the permutation-invariant version of the task reported by [26] who pretrained their models with a large amount of unlabeled samples.

As we have used the five-fold cross validation to find the optimal hyperparameters, we were able to use this to investigate the variance of the estimations of the $p$ values. Table 3 shows the averages and standard deviations of the estimated orders for MLPs trained on the five folds using the best hyperparameters. It is clear that in all the cases the orders ended up in a similar region near two without too much difference in the variance.

Similarly, we have trained five randomly initialized MLPs on MNIST and observed the similar phenomenon of all the resulting MLPs having similar distributions of the orders. The standard deviation of the averages of the learned orders was only 0.028 , while its mean is 2.16 .

The MLP having a single $L_{p}$ layer was able to classify the test samples of Pentomino with $31.38 \%$ error rate. This is the best result reported so far on Pentomino dataset [14] without using any kind of prior information about the task (the best previous result was $44.6 \%$ error).

On Forest Covertype an MLP having three $L_{p}$ layers was trained. The MLP was able to classify the test samples with only $2.83 \%$ error. The improvement is large compared to the previous state-of-the-art rate of $3.13 \%$ achieved by the manifold tangent classifier having four hidden layers of logistic sigmoid units 28. The result obtained with the $L_{p}$ is comparable to that obtained with the MLP having maxout units.

These results as well as previous best results for all datasets are summarized in Table 2 .

\footnotetext{
${ }^{1}$ Reported in 13 .

2 This result was obtained by training an MLP with rectified linear units which outperformed an MLP with maxout units.

3 Reported in [14.

4 This result was obtained by training an MLP with maxout units which outperformed an MLP with rectified linear units.
} 
In all experiments, we optimized hyperparameters such as an initial learning rate and its scheduling to minimize validation error, using random search [3, which is generally more efficient than grid search when the number of hyperparameters is not tiny. Each MLP was trained by stochastic gradient descent. All the experiments in this paper were done using the Pylearn2 library [12].

\subsection{Deep Recurrent Neural Networks}

We tried the polyphonic music prediction tasks with three music datasets; Nottingam, JSB and MuseData [7. The DOT-RNNs we trained had deep transition with $L_{p}$ units and tanh units and deep output function with maxout in the intermediate layer (see Fig. 6 for the illustration). We coarsely optimized the size of the models and the initial leaning rate as well as its schedule to maximize the performance on validation sets. Also, we chose whether to threshold the norm of the gradient based on the validation performance [25]. All the models were trained with dropout [16].

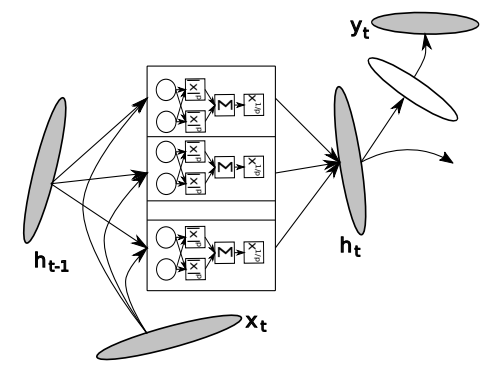

Fig. 6. The illustration of the DOT-RNN using $L_{p}$ units.

\begin{tabular}{c|cc}
\hline Fold & Mean & Std. Dev. \\
\hline 1 & 2.00 & $0.24 \times 10^{-4}$ \\
2 & 2.00 & $0.24 \times 10^{-4}$ \\
3 & 2.01 & $0.77 \times 10^{-4}$ \\
4 & 2.02 & $1.50 \times 10^{-4}$ \\
5 & 2.00 & $0.24 \times 10^{-4}$ \\
\hline
\end{tabular}

Table 3. The means and standard deviations of the estimated orders of $L_{p}$ units obtained during the hyperparameter search using the 5fold cross-validation.
As shown in Table 4, we were able to achieve the state-of-the-art results (RNN-only case) on all the three datasets. These results are much better than those achieved by the same DOT-RNNs using logistic sigmoid units in both deep transition and deep output, which suggests the superiority of the proposed $L_{p}$ units over the conventional saturating activation functions. This suggests that the proposed $L_{p}$ units are well suited not only to feedforward neural networks, but also to recurrent neural networks. However, we acknowledge that more investigation into applying $L_{p}$ units is needed in the future to draw more concrete conclusion on the benefits of the $L_{p}$ units in recurrent neural networks.

\section{Conclusion}

In this paper, we have proposed a novel nonlinear activation function based on the generalization of widely used pooling operators. The proposed nonlinear activation function computes the $L_{p}$ norm of several projections of the lower layer. Max-, average- and root-of-mean-squared pooling operators are special 


\begin{tabular}{c||cc|c}
\hline \multicolumn{1}{|c||}{} & \multicolumn{2}{|c|}{ DOT-RNN } & RNN \\
Dataset & $L_{p}$ & sigmoid & $*$ \\
\hline \hline Nottingam & 2.95 & 3.22 & 3.09 \\
JSB & 7.92 & 8.44 & 8.01 \\
Muse & 6.59 & 6.97 & 6.75
\end{tabular}

Table 4. The negative log-probability of the test sets computed by the trained DOT-RNNs. (*) These are the results achieved using DOT-RNNs having logistic sigmoid units, which we reported in 24]. (*) These are the previous best results achieved using conventional RNNs obtained in [2].

cases of the proposed activation function, and naturally the recently proposed maxout unit is closely related under an assumption of non-negative input signals.

An important difference of the $L_{p}$ unit from conventional pooling operators is that the order of the unit is learned rather than pre-defined. We claimed that this estimation of the orders is important and that the optimal model should have $L_{p}$ units with various orders.

Our analysis has shown that an $L_{p}$ unit defines a non-Euclidean subspace whose metric is defined by the $L_{p}$ norm. When projected back into the input space, the $L_{p}$ unit defines an ellipsoidal boundary. We conjectured and showed in a small scale experiment that the combination of these curved boundaries may more efficiently model separating curves of data with non-stationary curvature.

These claims were empirically verified via training both deep feedforward neural networks and deep recurrent neural networks. We tested the feedforward neural network on on four benchmark datasets; MNIST, Toronto Face Database, Pentomino and Forest Covertype, and tested the recurrent neural networks on the task of polyphonic music prediction. The experiments revealed that the distribution of the estimated orders of $L_{p}$ units indeed depends highly on dataset and is far away from a Dirac delta distribution. Additionally, our conjecture that deep neural networks with $L_{p}$ units will be able to achieve competitive generalization performance was empirically confirmed.

\section{Acknowledgments}

We would like to thank the developers of Pylearn2 12 and Theano 611. We would also like to thank CIFAR, and Canada Research Chairs for funding, and Compute Canada, and Calcul Québec for providing computational resources.

\section{References}

1. F. Bastien, P. Lamblin, R. Pascanu, J. Bergstra, I. J. Goodfellow, A. Bergeron, N. Bouchard, and Y. Bengio. Theano: new features and speed improvements. Deep Learning and Unsupervised Feature Learning NIPS 2012 Workshop, 2012.

2. J. Bayer, C. Osendorfer, D. Korhammer, N. Chen, S. Urban, and P. van der Smagt. On fast dropout and its applicability to recurrent networks. arXiv:1311.0701 [cs.NE], 2013.

3. J. Bergstra and Y. Bengio. Random search for hyper-parameter optimization. $J$. Machine Learning Res., 13:281-305, 2012. 
4. J. Bergstra, Y. Bengio, and J. Louradour. Suitability of V1 energy models for object classification. Neural Computation, 23(3):774790, Mar. 2011.

5. J. Bergstra, O. Breuleux, F. Bastien, P. Lamblin, R. Pascanu, G. Desjardins, J. Turian, D. Warde-Farley, and Y. Bengio. Theano: a CPU and GPU math expression compiler. In Proceedings of the Python for Scientific Computing Conference (SciPy), 2010.

6. J. Bergstra, O. Breuleux, F. Bastien, P. Lamblin, R. Pascanu, G. Desjardins, J. Turian, D. Warde-Farley, and Y. Bengio. Theano: a CPU and GPU math expression compiler. In Proceedings of the Python for Scientific Computing Conference (SciPy), June 2010. Oral Presentation.

7. N. Boulanger-Lewandowski, Y. Bengio, and P. Vincent. Modeling temporal dependencies in high-dimensional sequences: Application to polyphonic music generation and transcription. In ICML'2012, 2012.

8. Y. Boureau, J. Ponce, and Y. LeCun. A theoretical analysis of feature pooling in vision algorithms. In Proc. International Conference on Machine learning (ICML'10), 2010.

9. D. Ciresan, U. Meier, J. Masci, and J. Schmidhuber. Multi column deep neural network for traffic sign classification. Neural Networks, 32:333-338, 2012.

10. K. Fukushima. Neocognitron: A self-organizing neural network model for a mechanism of pattern recognition unaffected by shift in position. Biological Cybernetics, 36:193-202, 1980.

11. X. Glorot, A. Bordes, and Y. Bengio. Deep sparse rectifier neural networks. In AISTATS'2011, 2011.

12. I. J. Goodfellow, D. Warde-Farley, P. Lamblin, V. Dumoulin, M. Mirza, R. Pascanu, J. Bergstra, F. Bastien, and Y. Bengio. Pylearn2: a machine learning research library. arXiv preprint arXiv:1308.4214, 2013.

13. I. J. Goodfellow, D. Warde-Farley, M. Mirza, A. Courville, and Y. Bengio. Maxout networks. In ICML'2013, 2013.

14. C. Gulcehre and Y. Bengio. Knowledge matters: Importance of prior information for optimization. In International Conference on Learning Representations (ICLR'2013), 2013.

15. S. Haykin. Neural Networks and Learning Machines (3rd Edition). Prentice Hall, 3 edition, Nov. 2008.

16. G. E. Hinton, N. Srivastava, A. Krizhevsky, I. Sutskever, and R. Salakhutdinov. Improving neural networks by preventing co-adaptation of feature detectors. Technical report, arXiv:1207.0580, 2012.

17. D. Hubel and T. Wiesel. Receptive fields and functional architecture of monkey striate cortex. Journal of Physiology (London), 195:215-243, 1968.

18. A. Hyvärinen and U. Köster. Complex cell pooling and the statistics of natural images. Network: Computation in Neural Systems, 18(2):81-100, 2007.

19. K. Jarrett, K. Kavukcuoglu, M. Ranzato, and Y. LeCun. What is the best multistage architecture for object recognition? In Proc. International Conference on Computer Vision (ICCV'09), pages 2146-2153. IEEE, 2009.

20. A. Krizhevsky, I. Sutskever, and G. Hinton. ImageNet classification with deep convolutional neural networks. In Advances in Neural Information Processing Systems 25 (NIPS'2012). 2012.

21. Y. LeCun, L. Bottou, Y. Bengio, and P. Haffner. Gradient-based learning applied to document recognition. Proceedings of the IEEE, 86(11):2278-2324, Nov. 1998.

22. V. Nair and G. E. Hinton. Rectified linear units improve restricted Boltzmann machines. In L. Bottou and M. Littman, editors, Proceedings of the Twenty-seventh 
International Conference on Machine Learning (ICML-10), pages 807-814. ACM, 2010.

23. R. Pascanu and Y. Bengio. Revisiting natural gradient for deep networks. Technical report, arXiv:1301.3584, 2013.

24. R. Pascanu, C. Gulcehre, K. Cho, and Y. Bengio. How to construct deep recurrent neural networks. arXiv:1312.6026 [cs.NE], Dec. 2013.

25. R. Pascanu, T. Mikolov, and Y. Bengio. On the difficulty of training recurrent neural networks. In ICML'2013, 2013.

26. M. Ranzato, V. Mnih, J. M. Susskind, and G. E. Hinton. Modeling natural images using gated mrfs. IEEE Transactions on Pattern Analysis and Machine Intelligence, 35(9):2206-2222, 2013.

27. M. Riesenhuber and T. Poggio. Hierarchical models of object recognition in cortex. Nature Neuroscience, 1999.

28. S. Rifai, Y. Dauphin, P. Vincent, Y. Bengio, and X. Muller. The manifold tangent classifier. In NIPS'2011, 2011.

29. F. Rosenblatt. Principles of neurodynamics: perceptrons and the theory of brain mechanisms. Report (Cornell Aeronautical Laboratory). Spartan Books, 1962.

30. D. E. Rumelhart, G. E. Hinton, and R. J. Williams. Learning representations by back-propagating errors. Nature, 323:533-536, 1986.

31. J. Susskind, A. Anderson, and G. E. Hinton. The Toronto face dataset. Technical Report UTML TR 2010-001, U. Toronto, 2010.

32. M. Trebar and N. Steele. Application of distributed svm architectures in classifying forest data cover types. Computers and Electronics in Agriculture, 63(2):119 - 130, 2008 .

33. J. Yang, K. Yu, Y. Gong, and T. Huang. Linear spatial pyramid matching using sparse coding for image classification. In Proc. Conference on Computer Vision and Pattern Recognition (CVPR'2010), 2010. 